PREPARED FOR THE U.S. DEPARTMENT OF ENERGY, UNDER CONTRACT DE-AC02-76CH03073

PPPL-3913

PPPL-3913

UC-70

Double-kink Fishbone Instability Caused

by Circulating Energetic Ions

by

Ya.I. Kolesnichenko, V.V. Lutsenko,

V.S. Marchenko, and R.B. White

January 2004

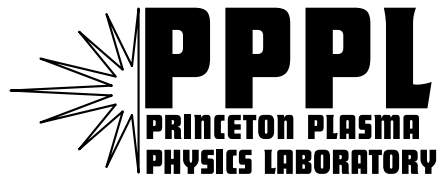

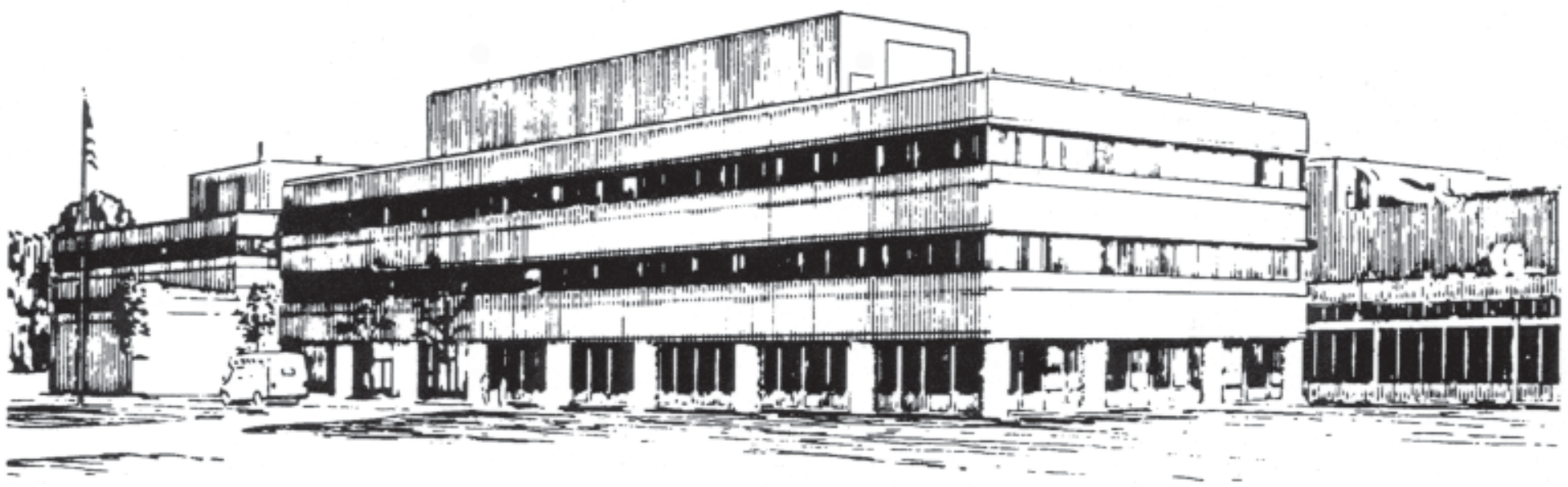

PRINCETON PLASMA PHYSICS LABORATORY PRINCETON UNIVERSITY, PRINCETON, NEW JERSEY 


\section{PPPL Reports Disclaimer}

This report was prepared as an account of work sponsored by an agency of the United States Government. Neither the United States Government nor any agency thereof, nor any of their employees, makes any warranty, express or implied, or assumes any legal liability or responsibility for the accuracy, completeness, or usefulness of any information, apparatus, product, or process disclosed, or represents that its use would not infringe privately owned rights. Reference herein to any specific commercial product, process, or service by trade name, trademark, manufacturer, or otherwise, does not necessarily constitute or imply its endorsement, recommendation, or favoring by the United States Government or any agency thereof. The views and opinions of authors expressed herein do not necessarily state or reflect those of the United States Government or any agency thereof.

\section{Availability}

This report is posted on the U.S. Department of Energy's Princeton Plasma Physics Laboratory Publications and Reports web site in Fiscal Year 2004. The home page for PPPL Reports and Publications is: http://www.pppl.gov/pub_report/

DOE and DOE Contractors can obtain copies of this report from:

U.S. Department of Energy

Office of Scientific and Technical Information

DOE Technical Information Services (DTIS)

P.O. Box 62

Oak Ridge, TN 37831

Telephone: (865) 576-8401

Fax: (865) 576-5728

Email: reports@adonis.osti.gov

This report is available to the general public from:

National Technical Information Service

U.S. Department of Commerce

5285 Port Royal Road

Springfield, VA 22161

Telephone: $1-800-553-6847$ or

(703) $605-6000$

Fax: (703) 321-8547

Internet: http://www.ntis.gov/ordering.htm 


\title{
Double-kink fishbone instability caused by circulating energetic ions
}

\author{
Ya.I. Kolesnichenko ${ }^{1}$, V.V. Lutsenko ${ }^{1}$, V.S. Marchenko ${ }^{1}$, R.B. White ${ }^{2}$ \\ ${ }^{1}$ Institute for Nuclear Research, Prospekt Nauky 47, Kyiv, 03680, Ukraine \\ 2 Princeton Plasma Physics Laboratory, \\ P.O. Box 451, Princeton, New Jersey, 08543, USA
}

(December 9, 2003)

\begin{abstract}
The destabilization of double kink modes by the circulating energetic ions in tokamaks with the plasma current having an off-axis maximum is studied. It is shown that the high-frequency fishbone instability [Energetic Particle Mode (EPM)] and the low-frequency (diamagnetic) fishbones are possible for such an equilibrium, their poloidal and toroidal mode numbers being not necessarily equal to unity. A new kind of the EPM instability, "doublet fishbones", is predicted. This instability is characterized by two frequencies; it can occur in a plasma with a non-monotonic radial profile of the energetic ions when the particle orbit width is less than the width of the region where the mode is localized. It is found that the diamagnetic fishbone branch exists even when the orbit width exceeds the mode width; in this case, however, the instability growth rate is relatively small.
\end{abstract}




\section{INTRODUCTION}

Fishbone instability was observed for the first time 20 years ago. ${ }^{1}$ It was characterized by the dominant mode numbers (both, poloidal, $m$, and toroidal, $n$ ) equal unity and resulted in strong loss of the injected ions. This instability was interpreted as an $m=$ $n=1$ rigid kink displacement of the plasma core with $r \leq r_{s}$, where $r_{s}$ is the radius of the $m / n$ surface. The calculated frequency was equal either to the precessional frequency of the energetic ions, $\omega_{D}$ [in which case the observations were explained by an Energetic Particle Mode (EPM) instability] or to the bulk-ion diamagnetic frequency, $\omega_{d i} \cdot{ }^{2,3}$ The mentioned instability was driven by the trapped energetic ions. Later a similar instability caused by the circulating ions was observed, and a theory of the destabilization of the $m=n=1$ rigid kink by circulating ions was suggested. ${ }^{4-6}$ Recently, experiments on the National Spherical Torus Experiment (NSTX) and a theoretical consideration have shown that fishbone activity can have $m \neq 1, n \neq 1$ dominant mode numbers. ${ }^{7}$ On the other hand, theory predicts that when $\beta$ is so high that the magnetic valley (a minimum of the equilibrium magnetic field) arises in the vicinity of the magnetic axis, the fishbone instabilities tend to be stabilized. ${ }^{8-10}$

The instabilities described above were relevant to plasmas with the monotonic profile of the safety factor, $q(r)$. On the the hand, it is known that regimes with non-monotonic $q(r)$ are characterized by improved plasma confinement and attract a considerable attention. Therefore, it is of interest to consider fishbone instabilities in plasmas with $q(r)$ having an off-axis minimum. A step in this direction was done in Ref. ${ }^{11}$, where it was shown that trapped energetic ions can destabilize a double kink mode. The considered instability is localized in the region $r_{s 1}<r<r_{s 2}$, where $r_{s 1}$ and $r_{s 2}$ are the $q=m / n \equiv q_{s}$ radii; it is characterized by a "top-hat" radial plasma displacement, $\xi_{r}(r) .{ }^{12}$ The destabilization of the double kink by the circulating ions is not studied yet. This issue is addressed in the present work.

As in Refs. ${ }^{12,11}$, we assume that $q(r)$ has a single off-axis minimum, $q_{\text {min }}$, and the mode width, $\Delta_{m}$, is small as compared to $r_{\text {min }}$, where $r_{\min }$ is determined by $q\left(r_{\min }\right)=q_{\min }$. 
The fishbone mode induced by the circulating particles exists due to energetic-ions finite orbit width, which provides a considerable energy exchange between the circulating particles and a kink perturbation. ${ }^{5,6}$ Therefore, an important parameter is the ratio of the orbit width, $\Delta r_{b}$, to the mode width, $\Delta_{m}$. In this work, we consider two limit cases, $\Delta_{m} \ll \Delta r_{b}$ and $\Delta r_{b} \ll \Delta_{m}$. In the latter case there are two characteristic frequencies, $\omega_{1} \sim k_{\|}^{\text {inner }} v_{\|}$and $\omega_{2} \sim k_{\|}^{\text {outer }} v_{\|}$, where $k_{\| 1}$ and $k_{\| 2}$ are characteristic longitudinal wave numbers near the inner edge and outer edge of the region where the mode is localized, respectively, $v_{\|}$is the velocity of the energetic ions along the magnetic field. A possibility of a "doublet" instability, i.e., the instability with $\omega \sim \omega_{1}$ and $\omega \sim \omega_{2}$ of a mode with given $m, n$ is considered in the work. The analysis is carried out in the assumption that the energetic ions have "standard" orbits with $\Delta r_{b} \ll r$.

The structure of the work is as follows. In the Sec. II A the equations describing both the high frequency fishbone branch and the low frequency branch in a plasma with the non-monotonic profile of the safety factor are formulated. These equations are analyzed for the cases of $\Delta_{m} \ll \Delta r_{b}$ and $\Delta_{m} \gg \Delta r_{b}$, in Sec. II B and Sec. II C, respectively. Section III contains the summary of the obtained results and a discussion of a possible relevance of the predicted "doublet" instability to experimental observations on the ASDEX-U tokamak. ${ }^{13}$

\section{FISHBONE ANALYSIS}

\section{A. Generic equations}

Let us assume first that $\omega \gg \omega_{d i}$. Then a generic form of the dispersion relation describing fishbone instabilities (and the influence of energetic ions on the ideal MHD instabilities) is

$$
\delta I+\delta W_{c}+\delta W_{h}=0
$$

where $\delta W_{c}$ is the MHD potential energy of the bulk plasma, $\delta W_{h}$ is the energy associated with the response of the energetic ions, and $\delta I$ is the sum of the kinetic and potential energies in two inertial layers at $r_{s 1}$ and $r_{s 2}$ given by (cf. ${ }^{12}$ ) 


$$
\begin{aligned}
& \delta I=-2 \pi^{2} \frac{R_{0}}{m^{2}} n_{0} m_{i} \int_{\text {layers }} r^{3}\left(\frac{d \xi_{r}}{d r}\right)^{2}\left[\omega^{2}-k_{\|}^{2} v_{A}^{2}\right] d r \\
& =-\frac{i \omega}{2 v_{A}} \frac{B^{2}}{|m| q_{s}}\left(r_{s 1}^{2}\left|s_{1}\right|+r_{s 2}^{2}\left|s_{2}\right|\right) \xi_{0}^{2}
\end{aligned}
$$

Here $\omega$ is the mode frequency (we assume $\omega>0), v_{A}$ is the Alfvén velocity, $k_{\|}=\left(m q^{-1}-\right.$ $n) / R_{0}$ is the longitudinal wave number, $R_{0}$ is the radius of the magnetic axis, $\xi_{0}$ is the amplitude of the radial component of the MHD displacement. The latter is taken in the form: $\vec{\xi}=\vec{\xi}_{0}(r) \exp [i(m \theta-n \phi-\omega t)]$, with $\vec{\xi}_{0}=\left(\xi_{0 r}, \xi_{0 \theta}\right), \xi_{0 r}(r)=\xi_{0} H(r-$ $\left.r_{\text {min }}+\Delta_{m} / 2\right) H\left(r_{\text {min }}+\Delta_{m} / 2-r\right), H(x)=\int_{-\infty}^{x} \delta(t) d t$, and $\xi_{0 \theta}$ is determined by the incompressibility condition $\nabla \cdot \vec{\xi}=0$.

It follows from Eqs. (1), (2) that the dispersion relation can be written in a form similar to that in the case of the monotonic $q(r)$ :

$$
i \frac{\omega}{\omega_{A}}+\lambda_{c}+\lambda_{h}=0
$$

where $\lambda_{c}$ and $\lambda_{h}$ are the normalized negatives of $\delta W_{c}$ and $\delta W_{h}$, respectively, $\omega_{A}$ is the Alfvén frequency. The fast ion response, $\lambda_{h}$, consists of the fluid (adiabatic) part, and the kinetic part: $\lambda_{h}=\lambda_{h f}+\lambda_{k}$.

The magnitudes in Eq. (3) can be written as follows (we put $r_{s 1} \approx r_{s 2} \approx r_{\text {min }}$, which is justified due to the assumption $\left.\Delta_{m} \ll r_{s}\right)$ : $11,12,14$

$$
\begin{gathered}
\omega_{A}=|m|\left(\left|s_{1}\right|+\left|s_{2}\right|\right) \frac{v_{A}}{q_{s} R_{0}} \\
\lambda_{c}=L \alpha^{2}\left(1-\frac{n q_{m i n}}{m}\right)^{1 / 2}-\frac{8}{15}\left(m^{2}-1\right)\left(1-\frac{n q_{m i n}}{m}\right)^{2} \\
-\frac{8 \pi r p^{\prime}}{B_{0}^{2}}\left(1-q_{m i n}^{2}\right)-\lambda_{\text {comp }}
\end{gathered}
$$

where $\alpha=-8 \pi R_{0} p^{\prime} q^{2} / B_{0}^{2}, p^{\prime} \equiv d p / d r, p$ is the plasma pressure, $L$ is a number that encapsulates the information regarding coupling to toroidally induced sideband displacements, ${ }^{12}$ $\lambda_{\text {comp }}$ is the stabilizing contribution associated with perpendicular compression; ${ }^{15}$

$$
\lambda_{h f}=-\frac{\pi^{2} m_{\alpha} q_{s}}{\xi_{0}^{2}\left(\left|s_{1}\right|+\left|s_{2}\right|\right)^{2} r_{m i n}^{2} B_{0}^{2}} \sum_{\sigma} \int d P_{\phi} \int v^{5} d v \int \frac{\partial F_{\alpha}}{\partial P_{\phi}} \tau_{b}\left\langle\xi_{0 r}^{2} r \cos \theta\right\rangle d \Lambda
$$




$$
\begin{aligned}
& \lambda_{k}=\frac{2 \pi^{2} R_{0} m_{\alpha} q_{s}^{2}}{\omega_{c \alpha} r_{\min }^{2} \xi_{0}^{2} B_{0}^{2}\left(\left|s_{1}\right|+\left|s_{2}\right|\right)^{2}} \sum_{\sigma} \int v^{3} d v \int d P_{\phi} \int d \Lambda \tau_{b} \frac{\partial F_{\alpha}}{\partial \mathcal{E}} \frac{\omega-n \omega_{* \alpha}}{\omega-k_{\|} v_{\|}} \\
& \times\left|\left\langle\left(\frac{v_{\perp}^{2}}{2}+v_{\|}^{2}\right) \vec{\xi} \cdot \vec{\kappa} \exp \left[i\left(\omega-k_{\|} v_{\|}\right) t\right]\right\rangle\right|^{2},
\end{aligned}
$$

where $\mathcal{E}=m_{\alpha} v^{2} / 2$ is the particle energy, $\Lambda \equiv \mu B_{0} / \mathcal{E}, P_{\phi}=m_{\alpha} v_{\|} R+(e / c) \psi$ is the canonical angular momentum, $\psi$ is the poloidal magnetic flux, $R$ is the distance from the major axis of the torus, $\omega_{* \alpha}=\left(\partial F_{\alpha} / \partial P_{\phi}\right)\left(\partial F_{\alpha} / \partial \mathcal{E}\right)^{-1}, \tau_{b}$ is the particle transit time, $\langle\ldots\rangle$ denotes the orbit averaging, $\vec{\kappa}=B^{-2} \nabla_{\perp}\left(B^{2} / 2+4 \pi p\right)$ is the field line curvature, $\sigma \equiv v_{\|} /\left|v_{\|}\right|, v_{\|}$and $v_{\perp}$ are the particle velocity along and across the magnetic field, respectively.

Taking into account that $\lambda_{c}$ does not depend on $\omega$ and assuming $\omega=\omega_{0}+i \gamma$ with $\gamma \ll \omega_{0}$, we can split Eq. (3) into two equations representing the real and imaginary parts of Eq. (3) as follows (the subscript "0" is omitted):

$$
\begin{aligned}
\frac{\gamma}{\omega_{A}} & =\left(\lambda_{c}+\operatorname{Re} \lambda_{h}\right)\left(1+\omega_{A} \frac{\partial \mathrm{I} m \lambda_{h}}{\partial \omega}\right)^{-1}, \\
& -\frac{\gamma \omega_{A}}{\omega} \frac{\partial \operatorname{Re} \lambda_{h}}{\partial \omega}=1+\frac{\omega_{A} \operatorname{I} m \lambda_{h}}{\omega} .
\end{aligned}
$$

Equations (8), (9) impose certain restrictions on $\lambda_{h}$. In particular, Eq. (8) with $\gamma \geq 0$ and $\omega_{A} \partial \mathrm{I} m \lambda_{h} / \partial \omega>-1$ is satisfied for a plasma on/below the ideal MHD stability limit $\left(\lambda_{c} \leq 0\right)$ only when $\operatorname{Re} \lambda_{h} \geq 0$. The first term in the RHS of Eq. (9) describes the continuum damping of the mode, whereas the second term drives the instability provided that $\operatorname{Im} \lambda_{h}<0$. It follows from Eq. (9) that $\partial \operatorname{Re} \lambda_{h} / \partial \omega>0$ for the instability to occur, which implies that the mode energy is negative, and thus Eq. (3) describes an EPM instability.

The sign of $\partial \mathrm{I} m \lambda_{h} / \partial \omega$ depends on the sign of $d p_{\alpha} / d r$ (with $p_{\alpha}$ the fast ion pressure) when the inhomogeneity term dominates in Eq. (7). Therefore, the physics of the destabilization of a double kink mode by the energetic ions with a non-monotonic $p_{\alpha}(r)$ and $\left|\omega_{A} \partial \mathrm{I} m \lambda_{h} / \partial \omega\right| \sim 1$ is more complicated than that in the case of a monotonic $p_{\alpha}(r)$.

When $\omega \sim \omega_{d i}$, the diamagnetic effects should to be taken into account. A corresponding dispersion relation for the monotonic $q(r)$ has been obtained in Ref. ${ }^{3}$ One can see that 
it has a similar form for the non-monotonic $q(r)$ when $\omega_{d i}\left(r_{s 1}\right)=\omega_{d i}\left(r_{s 2}\right)$, which can be justified in the considered case of $\Delta_{m} \ll r_{m i n}$ :

$$
\left[-\omega\left(\omega-\omega_{d i}\right)\right]^{1 / 2}=\omega_{A}\left[\lambda_{c}+\lambda_{h f}+\lambda_{k}(\omega)\right]
$$

where $\lambda_{c}+\lambda_{h f}+R e \lambda_{k} \geq 0$. The low-frequency (diamagnetic) fishbone branch is described by Eq. (10) in the case of

$$
\omega_{A}\left[\lambda_{c}+\lambda_{h}(\omega=0)\right] \ll 0.5 \omega_{d i}
$$

i.e., when the kink/tearing instability is stabilized by the plasma diamagnetism. Then Eq. (10) has the following fishbone solution obtained in the assumption $\left|\operatorname{Im} \lambda_{h}\right| \ll\left(\lambda_{c}+\right.$ $\left.\operatorname{Re} \lambda_{h}\right)$ :

$$
\omega \approx \omega_{d i}, \quad \gamma=-2 \omega_{d i}\left[\lambda_{c}+\operatorname{Re} \lambda_{h}\left(\omega_{d i}\right)\right] \operatorname{Im} \lambda_{h}\left(\omega_{d i}\right)
$$

It is clear that the energy associated with the energetic ions considerably depends on the $q(r)$ profile. When $q(r)$ is non-monotonic, there are two cases with different physics, $\Delta_{m}>\Delta r_{b}$ and $\Delta_{m}<\Delta r_{b}$, which will be considered below.

\section{B. The case of large orbit width}

Let us first carry out an analysis in the assumption of $\Delta_{m} \ll \Delta r_{b}$. We consider a plasma with a population of the energetic ions consisting only of the well-circulating particles. Then the equilibrium distribution function $F_{\alpha}$ can be approximated as follows:

$$
F_{\alpha}=\frac{\sqrt{2} m_{\alpha}^{3 / 2}}{\pi \mathcal{E}_{\alpha}} p_{\alpha}(\bar{r}) H\left(\mathcal{E}_{\alpha}-\mathcal{E}\right) \mathcal{E}^{-3 / 2} \delta(\Lambda)
$$

where $p_{\alpha}(\bar{r})$ is the beam particle pressure $\left[p_{\alpha}(\bar{r})=\int d^{3} v \mathcal{E} F_{\alpha}\right], \bar{r}$ is the orbit averaged radial coordinate of the particle. In addition, in this case we can put $v_{\|}(t)=$ const, $v_{\perp}(t)=$ const. This enables us to write $\vec{\xi}=\vec{\xi}_{0} \exp \left[-i\left(\omega-k_{\|} v_{\|}\right) t\right]$. Using this equation and omitting terms odd in $\theta$ we have:

$$
\left\langle\vec{\xi} \cdot \vec{\kappa} \exp \left[i\left(\omega-k_{\|} v_{\|}\right) t\right]\right\rangle=-\frac{1}{R_{0}}\left\langle\xi_{0 r} \cos \theta\right\rangle=\frac{\xi_{0}}{\pi R_{0}}\left(\sin \theta_{* 2}-\sin \theta_{* 1}\right),
$$


where $\theta_{* 1,2}$ are the poloidal angles at which a particle intersects the mode (see Fig. 1a), $\cos \theta_{*}=\left(\bar{r}-r_{\min } \pm \Delta_{m} / 2\right) / \Delta_{b}$, with $\Delta_{b}=\Delta r_{b} / 2$ (we used $\left.r(\theta)=\bar{r}-\Delta_{b} \cos \theta\right)$. Due to the small width of the mode we take into account only those particles, which intersect the mode region twice. The radial coordinate of these particles varies from $r>r_{s 2}$ to $r<r_{s 1}$ during the orbital motion, therefore for them

$$
\left(r_{\min }-\bar{r}\right)^{2}<\left(\Delta_{b}-0.5 \Delta_{m}\right)^{2}
$$

Then we can assume

$$
\left|r-r_{\min }\right| \Delta_{m} \ll \Delta_{b}^{2}-\left(r-r_{m i n}\right)^{2},
$$

which results in the following approximation of Eq. (14)

$$
\left\langle\vec{\xi} \cdot \vec{\kappa} \exp \left[i\left(\omega-k_{\|} v_{\|}\right) t\right]\right\rangle \approx \frac{\Delta_{m}}{\pi \Delta_{b}} \cot \hat{\theta}
$$

where $\hat{\theta}$ is defined by $\cos \hat{\theta}=\left(\bar{r}-r_{\min }\right) / \Delta_{b}$.

Putting Eqs. (17), (13) into Eq. (7), we obtain in the limit $\omega \ll n \omega_{* \alpha}$ :

$$
\lambda_{k}=-\frac{m}{2\left(\left|s_{1}\right|+\left|s_{2}\right|\right)^{2}}\left(\frac{\Delta_{m}}{r_{\min }}\right)^{2} \omega_{c \alpha} \int_{0}^{1} \frac{x d x}{\omega-\sigma k_{\|}(\bar{r}) v_{\alpha} x} \int \cot ^{2} \hat{\theta}(x, \bar{r}) \frac{d \beta_{\alpha}}{d \bar{r}} d \bar{r},
$$

where $x=v / v_{\alpha}$. The domain of two-dimensional integration in Eq. (18) is determined by Eq. (15) and $\Delta_{m} /\left(2 \Delta_{b \alpha}\right) \leq x$ with $\Delta_{\alpha}=\Delta\left(v=v_{\alpha}\right), v_{\alpha}=\sqrt{2 \mathcal{E}_{\alpha} / m_{\alpha}}$ [the latter condition follows from $\left.\Delta_{b}(v)>\Delta_{m}\right]$.

The imaginary part of $\lambda_{k}$ is associated with the resonance condition $\omega=k_{\|} v_{\|}$. Therefore, $\operatorname{Im} \lambda_{k} \neq 0$ only when $\sigma k_{\|}(\bar{r})>0$. One can see that $k_{\|}>0$ in the region $r_{s 1}<r<r_{s 2}$, where it has a maximum (for $m>0$ ), and $k_{\|}<0$ outside this region: this conclusion follows from the facts that $k_{\|}\left(r_{s 1}\right)=k_{\|}\left(r_{s_{2}}\right)=0$, and $d k_{\|} / d r=0, d^{2} q / d r^{2}>0$ at $r=r_{\text {min }}$. This implies that only the particles with $\bar{r}$ located inside the mode region interact with the mode when $v_{\|}>0$. However, for these particles $\cos \hat{\theta} \ll 1$ (because of the assumption $\left.\Delta_{m} \ll \Delta r_{b}\right)$. We conclude from here that strong energy exchange between the particles and the mode is possible only when $v_{\|}<0$, in which case $\cos \hat{\theta} \sim 1$ due to the particles in the regions $r<r_{s 1}, r>r_{s 2}$. 
In order to calculate the integrals in Eq. (18) analytically, we have to make some approximation for $k_{\|}$. Taking into account that $k_{\|}\left(r_{s 1}\right)=k_{\|}\left(r_{s 2}\right)=0$ and $d k_{\|} / d r=0$ at $r=r_{\min }$, we approximate $k_{\|}$as follows:

$$
k_{\|}=k_{\| \min } \frac{4\left(r-r_{\min }+\Delta_{m} / 2\right)\left(r_{\min }+\Delta_{m} / 2-r\right)}{\Delta_{m}^{2}} .
$$

Equation (19) is simplified in the region which mainly contributes to the integral:

$$
k_{\|} \approx-k_{\| \min } \frac{4\left(r-r_{\min }\right)^{2}}{\Delta_{m}^{2}}
$$

Using Eqs. (20), (18), (6), we obtain:

$$
\begin{aligned}
& \lambda_{k}=-\left.\frac{m H(-\sigma)}{8\left(\left|s_{1}\right|+\left|s_{2}\right|\right)^{2}}\left(\frac{\Delta_{m}}{r_{\text {min }}}\right)^{2} \frac{\omega_{c \alpha}}{k_{\| \min v_{\alpha}}}\left(\frac{\Delta_{m}}{\Delta_{\alpha}}\right)^{2} \Delta_{b \alpha} \frac{d \beta_{\alpha}}{d \bar{r}}\right|_{r_{\text {min }}} \\
& \times \int_{0}^{1} x d x \int_{\frac{\Delta_{m}}{2 \Delta_{\alpha}}-x}^{x-\frac{\Delta_{m}}{2 \Delta_{\alpha}}} \frac{u^{2} d u}{\left(\widetilde{\omega}-u^{2} x\right)\left(x^{2}-u^{2}\right)} \approx \frac{m H\left(-v_{\|}\right)}{24\left(\left|s_{1}\right|+\left|s_{2}\right|\right)^{2}} \frac{\Delta_{m}^{4}}{r_{m i n}^{2} \Delta_{\alpha}^{2}} \\
& \times\left.\left(\frac{\Delta_{\alpha}}{k_{\|} \rho_{\alpha}} \frac{d \beta_{\alpha}}{d \bar{r}}\right)\right|_{r_{m i n}}\left[\ln \frac{1+\sqrt{\widetilde{\omega}}}{1-\sqrt{\widetilde{\omega}}} \ln \frac{1+\sqrt{\widetilde{\omega}}}{\sqrt{\widetilde{\omega}}-1}+\ln \frac{4 \Delta_{\alpha}}{\Delta_{m}} \ln \left(1-\frac{1}{\widetilde{\omega}}\right)\right], \\
& \lambda_{h f}=-\left.\frac{2 q_{s}^{2} \Delta_{m}}{\left(\left|s_{1}\right|+\left|s_{2}\right|\right)^{2}} \frac{R}{r_{\min }} \frac{d \beta_{\alpha}}{d r}\right|_{r_{\text {min }}} \int_{0}^{1} d x \int_{\frac{\Delta_{m}}{\Delta r_{b \alpha}}-x}^{x-\frac{\Delta_{m}}{\Delta r_{b \alpha}}} \frac{u d u}{\sqrt{x^{2}-u^{2}}}=0,
\end{aligned}
$$

where $\widetilde{\omega} \equiv\left(\omega / k_{\| \min } v_{\alpha}\right)\left(\Delta_{m} / \Delta r_{b \alpha}\right)^{2}$.

An EPM instability has the frequency $\omega \lesssim k_{\|} v_{\|}$, i.e., $\widetilde{\omega} \lesssim 1$. Therefore, we can conclude from Eq. (21) that, when the radial profile of the energetic ions is monotonically decreasing, Re $\lambda_{k}<0$ (for all reasonable values of $\Delta r_{b \alpha} / \Delta_{m}$ ) and $\partial \operatorname{Im} \lambda_{k} / \partial \omega>0$. This means that a necessary condition for the existence of the EPM mode, Re $\lambda_{h}>0$ [see Eq. (8)] is not satisfied and, thus, the EPM fishbone mode is absent.

When $\omega \sim \omega_{d i}$, Eq. (10) should be used. Its fishbone solution is given by Eq. (12). After calculation of $\operatorname{Im} \lambda_{k}$ we obtain:

$$
\begin{aligned}
& \gamma=-\frac{\pi m q_{s}}{12\left(\left|s_{1}\right|+\left|s_{2}\right|\right)^{2}}\left[\lambda_{c}+\operatorname{Re} \lambda_{k}\left(\omega=\omega_{d i}\right)\right]\left(\frac{1}{k_{\|}} \frac{d \beta_{\alpha}}{d r}\right)_{\bar{r}=r_{m i n}} \\
& \times \frac{\omega_{A}^{2}}{\omega_{d i}} \frac{\Delta_{m}^{4}}{r_{m i n}^{2} \Delta_{b \alpha}^{2}} \ln \frac{4 \Delta_{b \alpha}}{\Delta_{m}} .
\end{aligned}
$$

This equation describes a low-frequency (diamagnetic) fishbone instability. The instability growth rate is rather small being proportional to small parameters $\Delta_{m}^{2} / r_{m i n}^{2}$ and $\Delta_{m}^{2} / \Delta_{b}^{2}$. 


\section{The case of small orbit width}

Now we assume that the energetic ion orbit width is small, $\Delta_{b} \ll \Delta_{m}$, but not vanishing: Finite magnitude of the orbit width plays an important role by providing the energy exchange between the well-circulating particles and a periodic perturbation with a "tophat" $\xi_{0}(r)$; only particles crossing the region of the mode localization during their orbital motion contribute to $\operatorname{I} m \lambda_{k}$. Because of the assumption $\Delta_{b} \ll \Delta_{m}$, the energetic ions crossing $r_{s 1}$ do not reach $r_{s 2}$ and vice versa, see Fig. 1b. This implies that the energetic ion response is actually the response calculated for the case of the $m=n=1$ rigid kink displacement in Ref. ${ }^{5}$, but with two rational surfaces. Therefore, we can easily generalize the result of Ref. ${ }^{5}$. We have:

$$
\lambda_{k}=\sum_{i=1,2} \lambda_{k i}=\sum_{i=1,2} \frac{1}{3} \frac{R_{0}}{r_{s i}} \frac{q_{s}^{2}}{\left(\left|s_{1}\right|+\left|s_{2}\right|\right)^{2}}\left[-\frac{\Delta r_{b \alpha}}{|s|} \frac{d \beta_{\alpha}}{d r}\right]_{r_{s i}} F\left(\frac{\omega}{\omega_{s i}}\right),
$$

where

$$
F(x) \equiv \frac{1}{\pi}\left\{10 x-8 x^{3 / 2}\left[\tan ^{-1} \frac{1}{\sqrt{x}}+\tanh ^{-1} \frac{1}{\sqrt{x}}\right]+\left(1+3 x^{2}\right) \ln \frac{1+x}{x-1}\right\}
$$

$\omega_{s i}=\left|s_{i}\right| v_{\| \alpha}^{2} /\left(\omega_{c} R_{0} r_{s i}\right)$. Note that $\omega_{s i} \sim \omega_{D}$, where $\omega_{D}$ is the precessional frequency of the well-trapped particles, when $s \sim q / 2$.

It follows from Eqs. (24), (25) that $\operatorname{Re} \lambda_{k}>0$ for $x>0$ and the monotonically decreasing profile of the energetic ions. Therefore, according to Eqs. (8), (9), in this case the system can be on the margin of stability $(\gamma=0)$ only when a plasma is below the ideal MHD limit $\left(\lambda_{c}<0\right)$. When $\lambda_{c}>0$, Eq. (8) predicts $\gamma>0$.

Below we carry out a more detailed stability analysis. We proceed from Eq. (3) valid for the arbitrary ratio of $\gamma / \omega$. Assuming $s_{1}=s_{2}, d \beta_{\alpha} /\left.d r\right|_{r_{s 1}}=d \beta_{\alpha} /\left.d r\right|_{r_{s 2}}$, and $v_{A}\left(r_{s 1}\right)=v_{A}\left(r_{s 2}\right)$, we write this equation as follows:

$$
0=D(\Omega)=-i \Omega-\tilde{\lambda}_{c}-\pi_{\alpha} F(\Omega)
$$

where $\tilde{\lambda}_{c}=\lambda_{c} \omega_{A} / \omega_{s 1}, \Omega=\omega / \omega_{s 1}$,

$$
\pi_{\alpha}=-\left.\frac{2}{3} \frac{m(m / n)^{2}}{|s|^{3}} \frac{v_{A}}{v_{\alpha}} R_{0} \frac{d \beta_{\alpha}}{d r}\right|_{r_{s}}
$$


The unstable solutions of the dispersion relation can be examined by a Nyquist analysis, see, e.g. ${ }^{16}$ Consider a contour in the $\Omega$ plane encircling the upper half plane, as shown in Fig. 2a. Its image under the map $D(\Omega)$ is presented in Fig. 2b. In this figure, when $\pi_{\alpha}$ exceeds a certain magnitude, $D(\Omega)$ encircles the coordinate origin, which corresponds to an unstable solution. The frequencies and the growth rates can be calculated from Eqs. (8) (9). The results presented in Fig. 3 shows that there are solutions with $\Omega \lesssim 1$ and, thus, an EPM instability is possible.

The found fishbone mode exists due to the resonance $\omega=k_{\|} v_{\|}$, which leads to one characteristic frequency $\left[\omega_{s} \sim k_{\|}^{\prime}\left(r_{s}\right) \Delta_{b} v_{\alpha}\right]$ when $k_{\|}^{\prime}\left(r_{s 1}\right)=k_{\|}^{\prime}\left(r_{s 2}\right)$. The latter is justified for $k_{\|}(r)$ symmetric with respect to $r_{\min }$ in the region $r_{s 1}<r<r_{s 2}$. However, in general, $s\left(r_{s 1}\right) \neq s\left(r_{s 2}\right)$ for a double kink mode with the finite width. For this reason, there are two, rather than one, characteristic frequencies. Therefore, one can expect that two fishbone instabilities with the frequencies of the order $\omega_{s 1} \equiv \omega_{s}\left(r_{s 1}\right)$ and $\omega_{s 2} \equiv \omega_{s}\left(r_{s 2}\right)$ and the same mode numbers $(m, n)$ will exist simultaneously under some conditions. Below we examine this possibility. We proceed from Eq. (3) written as

$$
0=D(\Omega)=-i \Omega-\tilde{\lambda}_{c}-\pi_{\alpha 1} F(\Omega)-\pi_{\alpha 2} F\left(\left|s_{1}\right| \Omega /\left|s_{2}\right|\right)
$$

where

$$
\pi_{\alpha i}=-\frac{8}{3} \frac{m(m / n)^{2}}{\left(\left|s_{1}\right|+\left|s_{2}\right|\right)^{2}}\left(\frac{v_{A}}{v_{\alpha}} \frac{R_{0}}{s} \frac{d \beta_{\alpha}}{d r}\right)_{r=r_{s i}} .
$$

The results of the Nyquist analysis for two particular cases are presented in Fig. 4. We observe that in both cases the curves encircle the origin of coordinate twice. This means that two instabilities co-exist, which we will refer to as a "doublet" instability. Their growth rates and the frequencies calculated as a function of $\pi_{\alpha 2}$ for $\left|s_{1} / s_{2}\right|=0.6$ and $\pi_{\alpha 1}=-2.5$ with the use of Eqs. (8), (9) are shown in Fig. 5. We observe that, first, there is no threshold with respect to $\pi_{\alpha 2}$ and, second, two instabilities with different frequencies exist for $\pi_{\alpha 2}$ in a certain interval (shaded region in Fig. 5). It is clear that one of the instabilities (with a lower frequency) arises mainly due to particles crossing $r_{s 1}$ when $\pi_{\alpha 2}$ is close to zero. Another instability, characterized by a higher frequency, arises when $\pi_{\alpha 2}$ 
exceeds the threshold magnitude $\pi_{\alpha 2}^{c r i t} \approx 2.4$. When $\pi_{\alpha 2}$ exceeds a certain magnitude, low frequency instability disappears. However, a more rigorous analysis is required to find the region where it disappears because the shown picture is based on the equations valid for $\gamma \ll \omega \lesssim \omega_{s}$. The Nyquist technique leads to $2.32<\pi_{\alpha 2}<2.45$. Thus, the region is rather narrow. On the other hand, calculations show that the region of the existence of the "doublet" instability is larger when a plasma is above the ideal MHD stability limit $\left(\lambda_{c}>0\right)$. For instance, we obtain $2.25<\pi_{\alpha 2}<2.47$ for $\lambda_{c}=0.1$ and the same other parameters.

Let us proceed to the low frequency range, $\omega \sim \omega_{d i} \ll \omega_{s}$. Using Eqs. (24), (12) we obtain:

$$
\gamma=-\left.\frac{4}{3} \frac{\omega_{A}^{2}}{\omega_{d i}}\left(\lambda_{c}+\lambda_{h f}\right) \frac{q_{s}^{2}}{\left(\left|s_{1}\right|+\left|s_{2}\right|\right)\left|s_{1} s_{2}\right|} \frac{R_{0}}{r_{\min }} \Delta_{b \alpha} \frac{d \beta_{\alpha}}{d \bar{r}}\right|_{r_{\text {min }}}
$$

Comparing Eq. (30) with Eq. (23) we conclude that the growth rate of the instability caused by the energetic ions with the narrow orbit width considerably exceeds the one associated with the wide-orbit-width particles.

\section{SUMMARY AND CONCLUSIONS}

In summary, we have developed a theory of the destabilization of the double kink mode by the circulating energetic ions through the resonance $\omega=k_{\|} v_{\|}$. It shows that both the EPM instability and low frequency fishbone instability $\left(\omega \approx \omega_{d i}\right)$ can be driven by the circulating beam ions in plasmas with the non-monotonic safety factor. The instability is localized between two rational surfaces, $r_{s 1}$ and $r_{s 2}$, with the same safety factor, $q_{s}\left(r_{s 1}\right)=q_{s}\left(r_{s 2}\right)=m / n$. A key parameter, which affects the instability growth rate, is the ratio of the orbit width of the energetic ions, $\Delta r_{b}$, to the mode width, $\Delta_{m}$. The instabilities are most strong when $\Delta r_{b} \ll \Delta_{m}$. In the contrary case, $\Delta r_{b} \gg \Delta_{m}$, the EPM instability is absent in plasmas with the $d \beta_{\alpha} / d r<0$.

A new kind of the instability, which we refer to as "doublet" fishbones, is predicted. This instability is characterized by two frequencies and two growth rates, although it is 
relevant to the same double kink mode. The ratio of the frequencies is about the ratio of the shears at two rational surfaces where the instability is localized. The instability can occur when the radial profile of the energetic ions has an off-axis maximum inside the region of the mode localization. The existence of the "double" fishbones is a consequence of the fact that there are two, rather that one, characteristic frequencies in the case of a non-monotonic $q(r): \omega_{1}=k_{\|}^{\text {inner }} v_{\|}$and $\omega_{2}=k_{\|}^{\text {outer }} v_{\|}$, where $k_{\|}^{\text {inner }}$ and $k_{\|}^{\text {outer }}$ are the characteristic longitudinal wave numbers relevant to the inner edge and outer edge of a double kink mode.

It seems possible that the "doublet" instability was observed in an ASDEX-U experiment reported in Ref. ${ }^{17}$ : In the mentioned experiment an $m=n=1$ mode with $f_{1} \sim 15$ $\mathrm{kHz}$ and $f_{2} \sim 20 \mathrm{kHz}$ (in the laboratory frame) was destabilized during tangential Neutral Beam Injection (NBI); The NBI power was deposited at about a half the plasma radius and, thus, $\beta_{\alpha}(r)$ was non-monotonic; there were two $q=1$ surfaces due to off-axis NBI current drive. Note that the observed doublet frequencies were comparable with the fishbone frequency during the radial injection, ${ }^{17}$ which is consistent with our theory as $\omega_{s} \sim \omega_{D}$. Moreover, observation of a continuous mode during tangential injection instead of bursting fishbones during the radial injection can be explained by weak redistribution of the circulating particles crossing $r_{s 1}$ and $r_{s 2}$ (in contrast to the resonant expulsion of the trapped particles). Of course, a more detailed analysis is required for the reliable interpretation of the experimental results.

\section{ACKNOWLEDGMENTS}

The research described in this publication was made possible in part by Award No. UP2-2419-KV-02 of the US Civilian Research \& Development Foundation and the Government of Ukraine, and U.S. Department of Energy Contract AC02-76CH03073. 


\section{REFERENCES}

${ }^{1}$ K. McGuire, R. Goldston, M. Bell et al., Phys. Rev. Lett. 50, 891 (1983).

${ }^{2}$ L. Chen, R.B. White, and M.N. Rosenbluth, Phys. Rev. Lett. 52, 1122 (1984).

${ }^{3}$ B. Coppi and F. Porcelli, Phys. Rev. Lett. 57, 2272 (1986).

${ }^{4}$ W.W. Heidbrink, K. Bol, D. Buchenauer, et al., Phys. Rev. Lett. 57, 835 (1986).

${ }^{5}$ R. Betti, J.P. Freidberg, Phys. Rev. Lett. 70, 3428 (1993).

${ }^{6}$ R. Betti, Plasma Phys. Controlled Fusion 35, 941 (1993).

${ }^{7}$ E.D. Fredrikson, L. Chen, and R.B. White, Nucl. Fusion 43, 1258 (2003).

${ }^{8}$ Ya.I. Kolesnichenko, V.V. Lutsenko, and V.S. Marchenko, Phys. Rev. Lett. 82, 3260 (1999).

${ }^{9}$ Ya.I. Kolesnichenko, V.V. Lutsenko, and V.S. Marchenko, Nucl. Fusion 40, 1731 (2000).

${ }^{10}$ Ya.I. Kolesnichenko, V.S. Marchenko, and R.B. White, Phys. Plasmas 8, 3143 (2001).

${ }^{11}$ P. Helander, C.G. Gimblett, R.J. Hastie, and K.G. McClements, Phys. Plasmas 4, 2181 (1997).

${ }^{12}$ C.G. Gimblett, R.J. Hastie, and T.S. Hender, Phys. Plasmas 3, 3369 (1996).

${ }^{13}$ W. Koependoerfer, C. Andelfinger, M. Ballico et al., in Proceedings of the 14th Conference on Plasma Heating and Controlled Fusion, Würzburg, 1992 (International Atomic Energy Agency, Vienna, 1993), Vol.I, p. 127.

${ }^{14}$ F. Porcelli, R. Stankiewicz, W. Kerner, and H.L. Berk, Phys. Plasmas 1, 470 (1994).

15 A. Bondeson and M.S. Chu, Phys. Plasmas 3, 3013 (1996).

${ }^{16}$ R.B. White "The Theory of Toroidally Confined Plasmas", Second Edition, Imperial College Press (2001).

${ }^{17}$ S. Günter, G. Gantenbein, A. Gude, V. Igochine, M. Maraschek, A. Mueck, S. Saarelma, 
O. Sauter, A.C.C. Sips, H. Zohm, and the ASDEX Upgrade Team, Nuclear Fusion 43, $161(2003)$. 


\section{FIGURES}
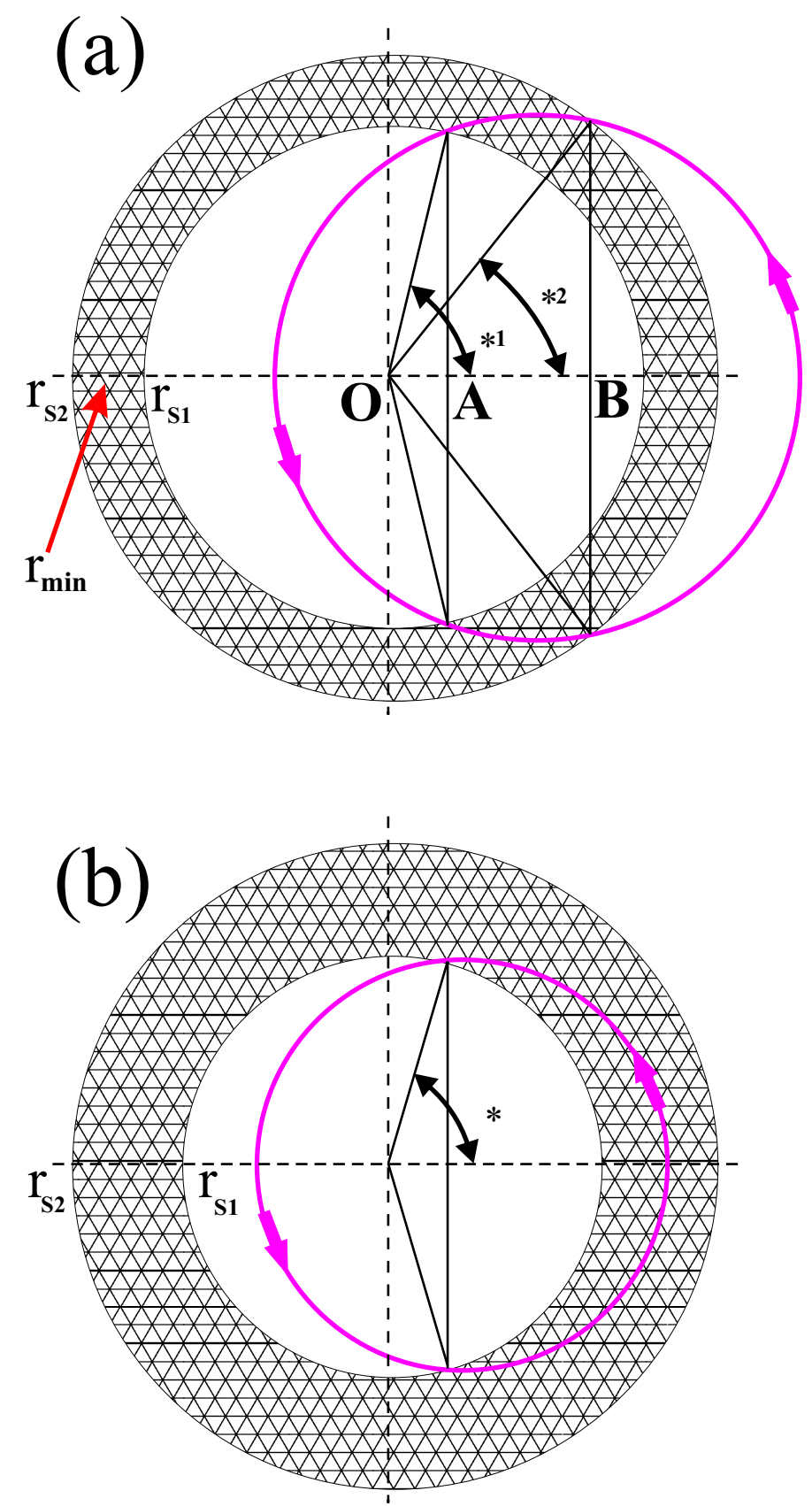

FIG. 1. A particle orbit crossing the region where a double kink mode is localized (shaded region): $a$, the orbit width exceeds the mode width; $b$, the mode width exceeds the orbit width. Notations: $r_{s 1}$ and $r_{s 2}$ are two rational surfaces with the same $q(r), r_{\min }$ is the radius where $q=q_{\text {min }}, \mathrm{OA}$ and $\mathrm{OB}$ are the cosines of the angles $\theta_{* 1}$ and $\theta_{* 2}$ at which a particle crosses the edges of the mode localization region. 

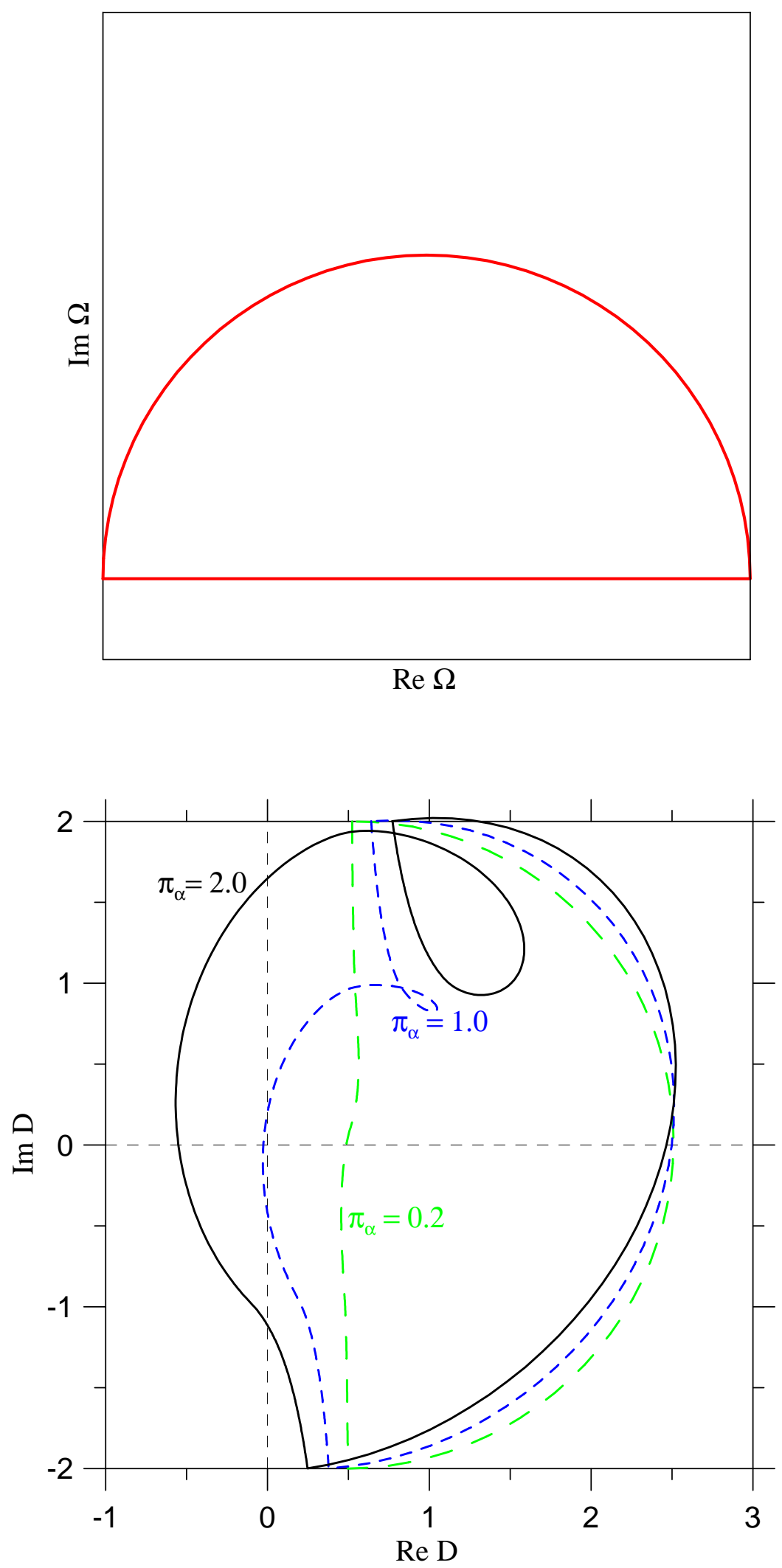

FIG. 2. Nyquist contour in the plane $(\operatorname{Re} \Omega, \operatorname{Im} \Omega)$ and its map in the plane $(\operatorname{Re} D, \operatorname{Im} D)$ when $s_{1}=s_{2}, \lambda_{c}=-0.5$ for various $\pi_{\alpha}$. Notations: $\Omega=\omega / \omega_{s 1}, \omega_{s 1}=\left|s_{1}\right| v_{\| \alpha}^{2} /\left(\omega_{c} R_{0} r_{s}\right)$, $\pi_{\alpha} \propto-d \beta_{\alpha} / d r$ 


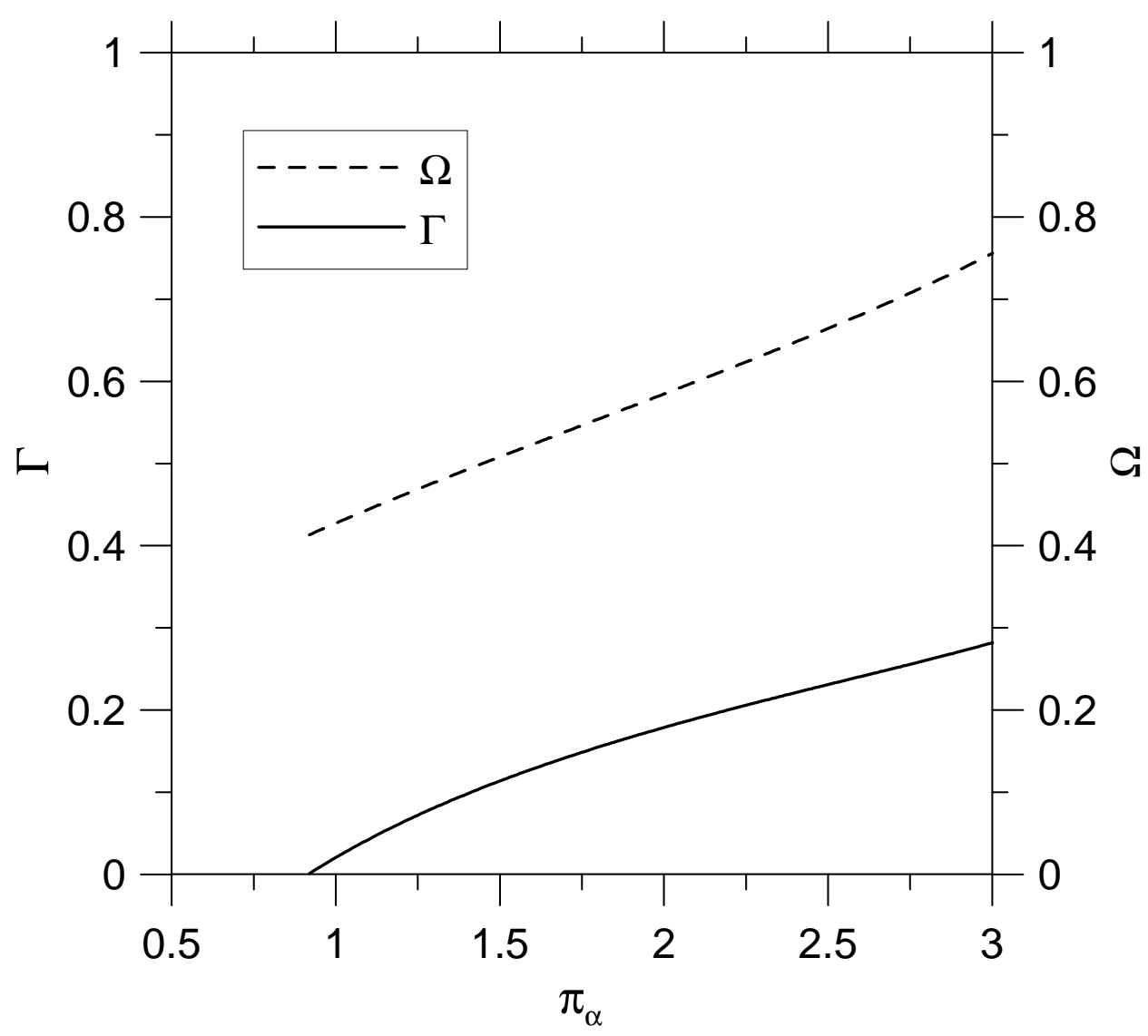

FIG. 3. Normalized growth rate, $\Gamma=\gamma / \omega_{s 1}$, and the mode frequency, $\Omega=\omega / \omega_{s 1}$, of the EPM fishbone instability in a plasma with a monotonic $\beta_{\alpha}(r)$ and $\widetilde{\lambda}_{c}=-0.1$. 


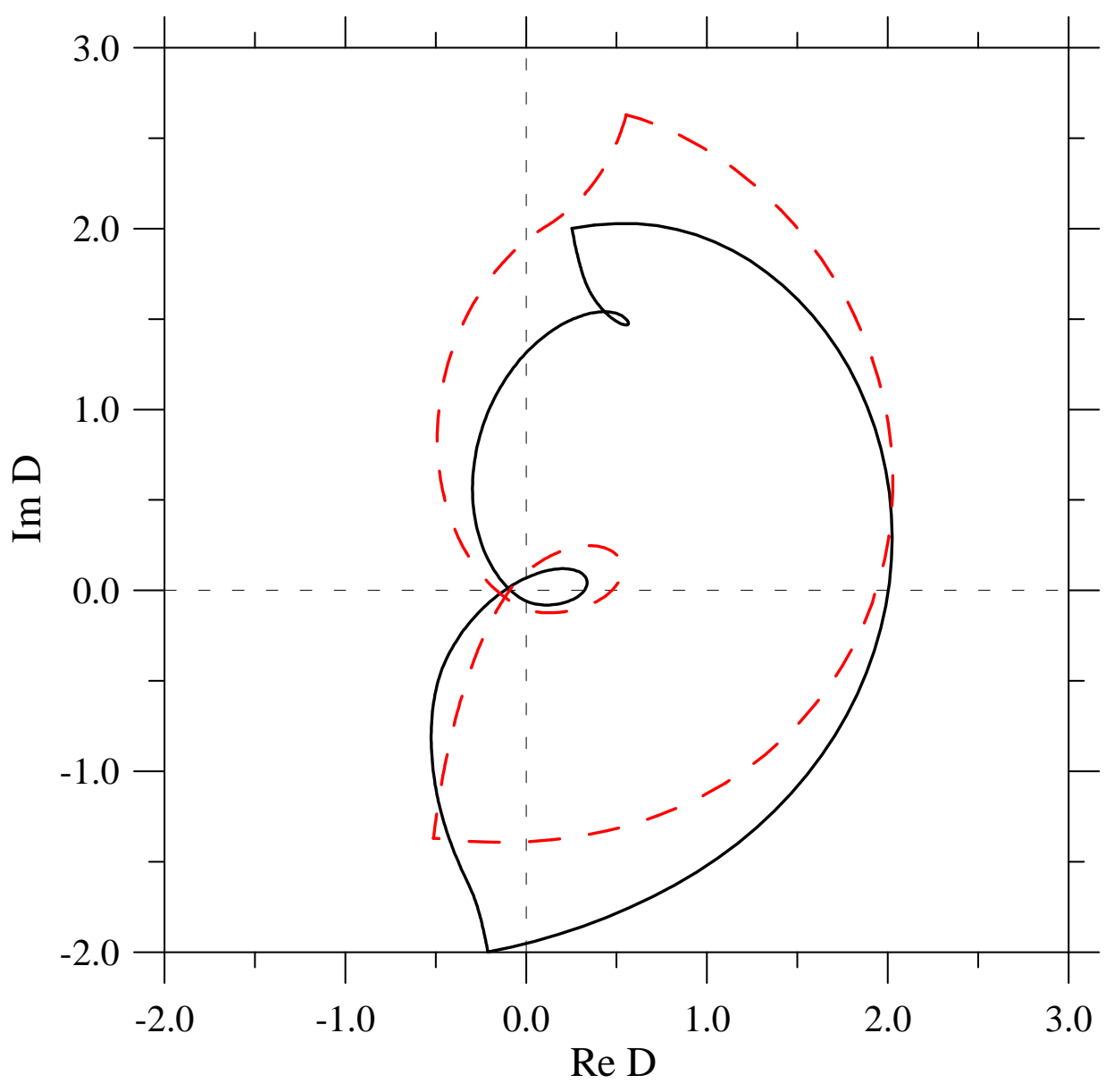

FIG. 4. Map of a Nyquist contour in the case of "doublet" instability for $\widetilde{\lambda}_{c}=-0.01$ : solid line, $s_{1} / s_{2}=0.6, \pi_{\alpha 1}=-2.5, \pi_{\alpha 2}=2.4$; dotted line, $s_{1} / s_{2}=0.2, \pi_{\alpha 1}=-1.5, \pi_{\alpha 2}=1.35$. 

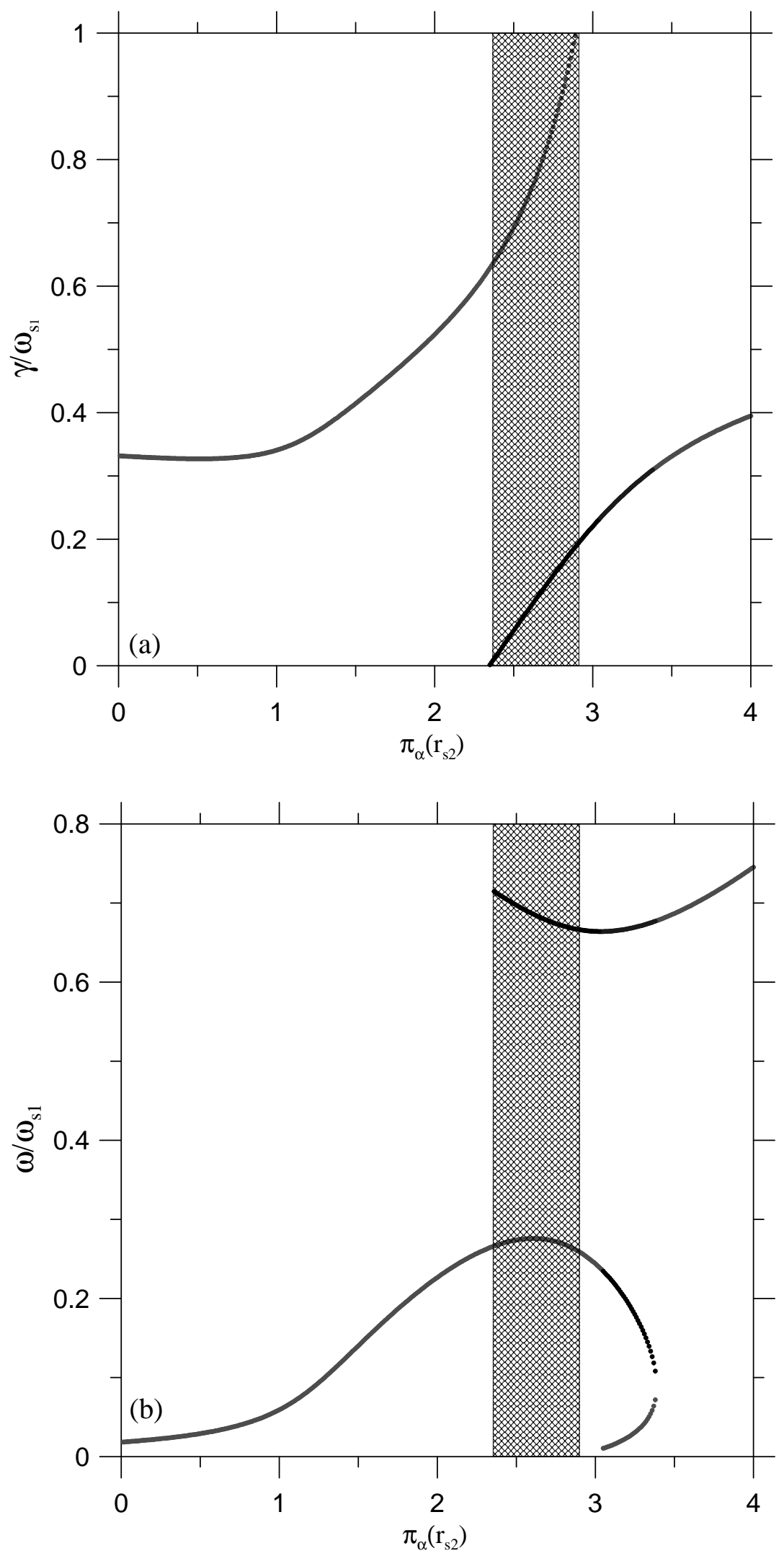

FIG. 5. The EPM fishbone instability in a plasma with a non-monotonic $\beta_{\alpha}(r)$ for $s_{1} / s_{2}=0.6, \pi_{\alpha 1}=-2.5$, and $\widetilde{\lambda}_{c}=-0.01: \mathrm{a}, \gamma / \omega_{s 1}$ versus $\pi_{\alpha}\left(r_{s 2}\right) ; \mathrm{b}, \omega / \omega_{s 1}$ versus $\pi_{\alpha}\left(r_{s 2}\right)$. Hatched region corresponds to a "doublet" instability. Its right edge obtained from the condition $\gamma=\omega_{s 1}$ is tentative. 


\section{External Distribution}

Plasma Research Laboratory, Australian National University, Australia

Professor I.R. Jones, Flinders University, Australia

Professor João Canalle, Instituto de Fisica DEQ/IF - UERJ, Brazil

Mr. Gerson O. Ludwig, Instituto Nacional de Pesquisas, Brazil

Dr. P.H. Sakanaka, Instituto Fisica, Brazil

The Librarian, Culham Laboratory, England

Mrs. S.A. Hutchinson, JET Library, England

Professor M.N. Bussac, Ecole Polytechnique, France

Librarian, Max-Planck-Institut für Plasmaphysik, Germany

Jolan Moldvai, Reports Library, Hungarian Academy of Sciences, Central Research Institute for Physics, Hungary

Dr. P. Kaw, Institute for Plasma Research, India

Ms. P.J. Pathak, Librarian, Institute for Plasma Research, India

Ms. Clelia De Palo, Associazione EURATOM-ENEA, Italy

Dr. G. Grosso, Instituto di Fisica del Plasma, Italy

Librarian, Naka Fusion Research Establishment, JAERI, Japan

Library, Laboratory for Complex Energy Processes, Institute for Advanced Study, Kyoto University, Japan

Research Information Center, National Institute for Fusion Science, Japan

Dr. O. Mitarai, Kyushu Tokai University, Japan

Dr. Jiangang Li, Institute of Plasma Physics, Chinese Academy of Sciences, People's Republic of China

Professor Yuping Huo, School of Physical Science and Technology, People's Republic of China

Library, Academia Sinica, Institute of Plasma Physics, People's Republic of China

Librarian, Institute of Physics, Chinese Academy of Sciences, People's Republic of China

Dr. S. Mirnov, TRINITI, Troitsk, Russian Federation, Russia

Dr. V.S. Strelkov, Kurchatov Institute, Russian Federation, Russia

Professor Peter Lukac, Katedra Fyziky Plazmy MFF UK, Mlynska dolina F-2, Komenskeho Univerzita, SK-842 15 Bratislava, Slovakia

Dr. G.S. Lee, Korea Basic Science Institute, South Korea

Institute for Plasma Research, University of Maryland, USA

Librarian, Fusion Energy Division, Oak Ridge National Laboratory, USA

Librarian, Institute of Fusion Studies, University of Texas, USA

Librarian, Magnetic Fusion Program, Lawrence Livermore National Laboratory, USA

Library, General Atomics, USA

Plasma Physics Group, Fusion Energy Research Program, University of California at San Diego, USA

Plasma Physics Library, Columbia University, USA

Alkesh Punjabi, Center for Fusion Research and Training, Hampton University, USA

Dr. W.M. Stacey, Fusion Research Center, Georgia Institute of Technology, USA

Dr. John Willis, U.S. Department of Energy, Office of Fusion Energy Sciences, USA

Mr. Paul H. Wright, Indianapolis, Indiana, USA 
The Princeton Plasma Physics Laboratory is operated by Princeton University under contract with the U.S. Department of Energy.

\author{
Information Services \\ Princeton Plasma Physics Laboratory \\ P.O. Box 451 \\ Princeton, NJ 08543
}

Phone: 609-243-2750

Fax: 609-243-2751

e-mail: pppl_info@pppl.gov

Internet Address: http://www.pppl.gov 